\title{
ON A GENERALIZATION OF THE GAMMA FUNCTION AND ITS APPLICATION TO CERTAIN DIRICHLET SERIES
}

\author{
BY CHUNG-MING AN ${ }^{1}$
}

\author{
Communicated by G. D. Mostow, December 9, 1968
}

In this paper, we will introduce certain Dirichlet series which generalize Epstein's Zeta function. We prove that these Dirichlet series possess analytic continuation as meromorphic functions. In obtaining the analytic continuation, we introduce certain integrals which generalize the Gamma function and establish the analytic property of these integrals.

We consider Dirichlet series of the type

$$
\zeta(s)=\sum_{\gamma \in Z^{n}-N_{F}} h(\gamma) F(\gamma)^{-82}
$$

where $F(X) \in R[X]$ is a polynomial of degree $d(>0), \quad X$ $=\left(X_{1}, \cdots, X_{n}\right)$, such that $F_{d}(X)$, the highest homogeneous part of $F(X)$, vanishes only at the origin, $N_{F}=\left\{x \in R^{n} \mid F(x)=0\right\}$; and $h(x)$ is in $S P\left(R^{n}\right)$ which is the smallest subring of $C^{\infty}\left(R^{n}\right)$ containing $R[X]$ and $S\left(R^{n}\right)$, the Schwartz space. It is easy to see that $S P\left(R^{n}\right)$ is the ring containing all functions $h(x)$ of the form $h(x)=f(x)+G(x)$ where $f(x) \in S\left(R^{n}\right)$ and $G(X) \in R[X]$.

I wish to take this opportunity to express my gratitude to Professor Takashi Ono for his valuable suggestions, direction and encouragement in the preparation of this work.

1. We write

$$
F(X)=F_{d}(X)+\cdots+F_{0}(X)
$$

where $F_{q}(X), q=0,1, \cdots, d$, are homogeneous parts of $F(X)$ of degree $q$. For $x=\left(x_{1}, \cdots, x_{n}\right) \in R^{n}$, we put $|x|=\left(x_{1}^{2}+\cdots+x_{n}^{2}\right)^{1 / 2}$. Let $S^{n-1}=\left\{x \in R^{n}|| x \mid=1\right\}$.

We shall consider the following three conditions:

(A) $N_{F}$ is compact,

(B) $N_{\boldsymbol{F}_{d}}$ is compact,

(C) $F(X)$ is homogeneous and $N_{F}$ is compact.

1 The results announced here are contained in the author's doctoral thesis written under the guidance of Professor Takashi Ono at the University of Pennsylvania.

${ }^{2}$ For subsets $A, B$ in a set, $A-B$ denotes the set of points in $A$ but not in $B$. 
It is clear that $(C) \Rightarrow(B) \Rightarrow(A)$. The following lemma is easy to prove by using [3, Lemma 1$]$.

LEMMA 1. If (A) holds, then there are positive constants $c_{1}, \beta, K$ such that

$$
|F(x)| \geqq c_{1}|x|^{-\beta}, \quad \text { for }|x| \geqq K .
$$

We shall prove the following

THEOREM 1. If (A) holds, the integral, for suitable $K$,

$$
J_{2}(s)=\int_{\|x\|_{Z} K} f(x) F(x)^{8} d x, \quad f(x) \in S\left(R^{n}\right),{ }^{3}
$$

represents an entire function of $s$, where $s=\sigma+t i$ is a complex number and $\|x\|=\max _{1 \leq i \leq n}\left(\left|x_{i}\right|\right)$.

Proof. It is sufficient to show that the integral

$$
J_{2}^{\prime}(s)=\int_{|x| \geq K} f(x) F(x)^{s} d x
$$

converges absolutely. We shall do for $\sigma \geqq 0$ and $\sigma \leqq 0$, separately.

(a) Assume $\sigma \geqq 0$. It is obvious that there is a constant $c>0$ such that $|F(x)| \leqq c|x|^{d}$ and for an arbitrary integer $k$, there is a constant $M$ (depending only on $f(x)$ and $k$ ) such that

$$
|f(x)| \leqq M /|x|^{k+n} \text {. }
$$

Hence we have, by using the polar coordinates in $n$-dimensions, i.e. $d x=r^{n-1} d r d \omega$,

$$
\int_{|x| \geq K}\left|f(x) F(x)^{s}\right| d x \leqq M c^{\sigma} e^{\pi|t|} \int_{S^{n-1}} d \omega \int_{K}^{\infty} r^{d \sigma-k-1} d r .
$$

The integral exists for $d \sigma-k<0$, i.e. $\sigma<k / d$. Since $k$ can be arbitrarily large, we see that $J_{2}^{\prime}(s)$ converges absolutely for $\sigma \geqq 0$.

(b) Assume $\sigma \leqq 0$. By Lemma 1, we have

$$
\int_{|x| \geq K}\left|f(x) F(x)^{\bullet}\right| d x \leqq M c_{1}^{\sigma} e^{\pi|t|} \int_{S^{n-1}} d \omega \int_{K}^{\infty} r^{-\beta \sigma-k-1} d r .
$$

The integral exists for $-\beta \sigma-k<0$, i.e. $\sigma>-k / \beta$. As in (a), we see that $J_{2}^{\prime}(s)$ converges absolutely for $\sigma \leqq 0$.

${ }^{3}$ For complex numbers $a, s, a^{*}=\exp [s(\log |a|+\arg (a))]$. 
2. Theorem 2. If $(\mathrm{C})$ holds for $F(X)$, then the integral

$$
J(s)=\int_{R^{n}} f(x) F(x)^{8} d x, \quad f(x) \in S\left(R^{n}\right),
$$

converges absolutely for $\sigma>-n / d$, and hence it represents a regular function in the half-plane. Moreover, it can be analytically continued into the whole s-plane as a meromorphic function with poles possible only for $s=-(n+h) / d, h=0,1,2, \cdots$, these poles biing simple if present.

Proof. We write, for suitable $K$ as in Theorem 1,

$$
J(s)=J_{1}(s)+J_{2}(s)
$$

where

$$
J_{1}(s)=\int_{|x| \leq K} f(x) F(x)^{\bullet} d x, \quad J_{2}(s)=\int_{|x| \geq K} f(x) F(x)^{\bullet} d x .
$$

By Theorem 1, we see that $J_{2}(s)$ is an entire function. We have

$$
J_{1}(s)=\int_{s^{n-1}} F(\omega) \cdot \int_{0}^{K} f(r \omega) r^{n+d \sigma-1} d r d \omega .
$$

Hence it is enough to show that the integral

$$
J_{1}^{*}(s)=\int_{0}^{K} f(r \omega) r^{n+d s-1} d r
$$

converges absolutely for each $\omega$ if $n+d \sigma>0$. By integration by parts, we have

$$
J_{1}^{*}(s)=\left[\frac{1}{n+d s} f(r \omega) r^{n+d s}\right]_{0}^{K}-\frac{1}{n+d s} \int_{0}^{K} \frac{\partial f(r \omega)}{\partial r} r^{n+d s} d r .
$$

The integral on the right side converges absolutely for $n+d \sigma>0$. We can easily see that the integral $J_{1}^{*}(s)$ can be analytically continued into the half-plane $\sigma>-(n+1) / d$ and has at most simple poles at $s$ $=-n / d$, and so $J_{1}(s)$ has the same property. Since $f(r \omega)$ has infinitely many derivatives with respect to $r$, we may use integration by parts infinitely many times. The analytic continuation of $J_{1}(s)$ into the whole $s$-plane can be obtained by this method, q.e.d.

REMARK 1. Let $n=1, f(x)=e^{-x^{2}}$ and $F(X)=X^{2}$. Then the Gamma function $\Gamma(s)=J\left(s-\frac{1}{2}\right)$.

REMARK 2. The residue of $J(s)$ at $s=-n / d$ is 


$$
\frac{1}{d} f(0) \int_{s^{n-1}} F(\omega)^{-n / d} d \omega
$$

because

$$
\begin{aligned}
\operatorname{Res} J(s)= & \operatorname{Res}_{s=-n / d} J_{1}(s)=\int_{s^{n-1}}\{ \\
& \frac{1}{d} f(K \omega) \\
& \left.-\frac{1}{d} \int_{0}^{K} \frac{\partial f(r \omega)}{\partial r} d r\right\} F(\omega)^{-n / d} d \omega .
\end{aligned}
$$

Hence, $J(s)$ is regular at $s=-n / d$ if $f(0)=0$ or

$$
\int_{s^{n-1}} F(\omega)^{-n / d} d \omega=0 \text {. }
$$

REMARK 3. If a polynomial $F(X)$ is such that the corresponding hypersurface is nonsingular, T. Ono [6] has proved that the integral $J(s)$ converges absolutely for $\sigma>-1$.

3. Let $G(X) \in R[X]$ be a polynomial of degree $p$. We shall follow Mahler's method [5] to prove the following theorem.

THEOREM 3. If (B) holds, the integral, with suitable $K$,

$$
I(s)=\int_{\|x\|_{\geq K}} G(x) F(x) \cdot d x
$$

converges absolutely for $\sigma<-(n+p) / d$, and hence it represents a regular function in this half-plane. Moreover, it can be analytically continued into the whole s-plane as a meromorphic function with poles possible only for

$$
s=-\frac{n+p-h}{d}, \quad\left(\begin{array}{l}
h=0, \quad 1, \quad 2, \cdots \\
s \neq 0,-1,-2, \cdots
\end{array}\right),
$$

these poles being simple if present. Its residue at $s=-(n+p) / d$ equals

$$
\lambda_{p}=-\frac{1}{d} \int G_{p}(\omega) F_{d}(\omega)^{-(n+p) / d} d \omega .
$$

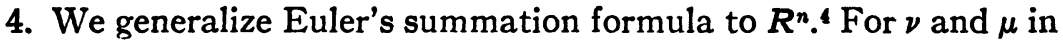
$Z_{+}$and $x$ in $R$, we put $h_{\nu}^{(\mu)}(x)=(-1)^{\nu} B_{\nu} / \nu !$ for $\nu=0,1, \cdots, \mu-1$,

4In Mahler's paper [5], a generalization of Euler's summation formula is given. Our generalization in this paper is more general than his. 
and $h_{\mu}^{(\mu)}(x)=\left((-1)^{\mu-1} /(\mu-1) !\right) \sigma_{\mu}(x)$ where $B$, is the $\nu$ th Bernoulli number and $\sigma_{\mu}(x)$ is the $\mu$ th Bernoulli polynomial. For $k=\left(k_{1}, \cdots, k_{n}\right)$ and $q=\left(q_{1}, \cdots, q_{n}\right)$ in $Z_{+}^{n}$ and $x$ in $R^{n}$, we put

$$
h_{q}^{(k)}(x)=h_{q_{1}}^{\left(k_{1}\right)}\left(x_{1}\right) \cdots h_{q_{n}}^{\left(k_{n}\right)}\left(x_{n}\right)
$$

and

$$
H_{q}(x)=\frac{\partial^{|q|^{+}}}{\partial x_{1}^{q_{1}} \cdots \partial x_{n}^{q_{n}}} H(x)
$$

where $H(x) \in C^{\infty}\left(R^{n}\right)$ and $|q|^{+}=q_{1}+\cdots+q_{n}$. It is easy to verify the following lemma.

LEMma 2. If the integral $\int_{\|x\| \geqq K} H_{q}(x) d x$ converges absolutely for all $q \in \boldsymbol{Z}_{+}^{n}$, then for any $k \in \boldsymbol{Z}_{+}^{n}$, we have

$$
\sum_{\gamma \in Z^{n}}^{\prime} H(\gamma)=\sum_{q_{1}=0}^{k_{1}} \cdots \sum_{q_{n}=0}^{k_{n}} \int_{\|x\|_{\geq K}} h_{q}^{(k)}(x) H_{q}(x) d x
$$

where $\sum^{\prime}$ means to sum over all integral points $\gamma$ with $\gamma_{i} \notin(-K, K]$ for at least one $i$.

5. Let $H(x)=f(x) F(x)^{-s}, f(x)$ and $F(X)$ be as in Theorem 1. We shall prove

Theorem 4. If $(A)$ holds, then the infinite series

$$
\zeta(s)=\sum_{\gamma \in Z^{n}-N_{F}} H(\gamma)
$$

represents an entire function of $s$.

Proof. The following formula is easily proved by induction on $|q|^{+}$, whenever $H_{q}(x)$ exists:

$$
H_{q}(x)=\sum_{h=0}^{|q|^{+}}\left[\begin{array}{r}
-s \\
h
\end{array}\right] \phi_{q}^{(h)}(x) F(x)^{-s-h}
$$

where

$$
\left[\begin{array}{r}
-s \\
h
\end{array}\right]=\left(\begin{array}{r}
-s \\
h
\end{array}\right) h !
$$

and $\phi_{Q}^{n}$ is in $S\left(R^{n}\right)$. Let $k_{1}=\cdots=k_{n}=1$. By Theorem 1 and Lemma 2, we obtain the theorem, q.e.d. 
6. Let $H(x)=G(x) F(x)^{-8}, G(X)$ and $F(X)$ be as in Theorem 2. We shall prove

THEOREM 5. If (B) holds, then the infinite series

$$
\zeta(s)=\sum_{\gamma \in Z^{n}-N_{F}} H(\gamma)
$$

represents a regular function for $\sigma>(n+p) / d$ and can be analytically continued into the whole s-plane as a meromorphic function with poles possible only for

$$
s=\frac{n+p-h}{d}, \quad\left(\begin{array}{l}
h=0, \quad 1, \quad 2, \cdots \\
s \neq 0,-1,-2, \cdots
\end{array}\right)
$$

these poles being simple if present. Its residue at $s=(n+p) / d$ equals $-\lambda_{p}$, where $\lambda_{p}$ is given in Theorem 3.

Proof. Again, we can prove the following formula by induction on $|q|^{+}$, whenever $H_{q}(x)$ exists:

$$
H_{q}(x)=\sum_{h=0}^{|q|^{+}}\left[\begin{array}{r}
-s \\
h
\end{array}\right] \psi_{q}^{(h)}(x) F(x)^{-8-h}
$$

where $\psi_{q}^{(h)}(x) \in R[X]$ is a polynomial of degree $p+d h-|q|+$ which is independent of $s$; the polynomial is 0 if $p+d h-|q|+<0$. By this formula, Lemma 2 and Theorem 2, we obtain the theorem, q.e.d.

7. Combining all those results, we obtain the following

MaIn Theorem. Let

$$
\zeta(s)=\sum_{\gamma \in Z^{n}-N_{F}} h(\gamma) F(\gamma)^{-s}, \quad h(x) \in S P\left(R^{n}\right) .
$$

(a) Assume that (B) holds for $F(X)$ and $G(X) \not \equiv 0$, then $\zeta(s)$ is regular for $\sigma>(n+p) / d$ and can be analytically continued into the whole s-plane as a meromorphic function with poles possible only for

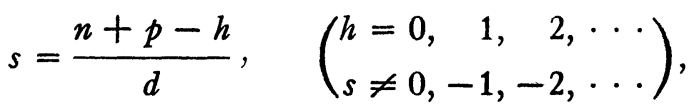

these poles being simple if present. Its residue at $s=(n+p) / d$ equals $-\lambda_{p}$ where $\lambda_{p}$ is given in Theorem 3.

(b) Assume that (A) holds for $F(X)$ and $G(X) \equiv 0$, then $\zeta(s)$ is an entire function. 


\section{BIBLIOGRAPHY}

1. E. Artin, The gamma function, Holt, New York, 1964.

2. Philip J. Davis and Philip Rabinowitz, Numerical integration, Blaisdell, Waltham, Mass., 1967.

3. L. Hörmander, On the division of distribution by polynomials, Ark. Mat. 3 (1958), 555-568.

4. K. Knopp, Theory and application of infinite series, Blachie, London, 1951.

5. K. Mahler, Über einer Satz von Mellin, Math. Ann. 100 (1928), 384-395.

6. T. Ono, An integral attached to a hypersurface, Amer. Math. J. (to appear).

University of Pennsylvania, Philadelphia, Pennsyluania 19104

\section{FUNCTIONS OF BOUNDED CONVEXITY}

BY A. WAYNE ROBERTS AND DALE E. VARBERG ${ }^{1}$

Communicated by R. Creighton Buck, October 31, 1968

1. Introduction. Functions of bounded variation on $[a, b]$ are those functions for which

$$
V_{a}^{b}(f)=\operatorname{Sup}_{\boldsymbol{P}} V(f, P)=\operatorname{Sup}_{\boldsymbol{P}} \sum_{j=1}^{n}\left|\Delta f_{j}\right|
$$

is finite. An important theorem about the set $B V[a, b]$ of all such functions says that this set may be characterized as the set of all functions representable as the difference of two nondecreasing functions. Stated with less precision but more suggestion for our purposes, $B V[a, b]$ is the set of all functions representable as the difference of two functions with nonnegative first derivatives. It is then natural to consider the set of all functions representable as the difference of two functions with nonnegative second derivatives (convex functions, roughly speaking).

We begin our study with an expression that plays the role of (1). For a partition $P=\left\{a=x_{1}<x_{2}<\cdots<x_{n}=b\right\}$, let $\square f_{j}$ $=\left[f\left(x_{j}\right)-f\left(x_{j-1}\right) /\left(x_{j}-x_{j-1}\right)\right]$.

Definition 1. For $f:[a, b] \rightarrow R$, let

$$
K_{a}^{b}(f)=\operatorname{Sup}_{\boldsymbol{P}} K(f, P)=\operatorname{Sup}_{\boldsymbol{P}} \sum_{j=1}^{n-1}\left|\square f_{j+1}-\square f_{j}\right| \text {. }
$$

1 The second author was supported by the National Science Foundation under grant number GP-7843. 